\title{
Incidence and risk factors of neonatal hypothermia at referral hospitals in Tehran, Islamic Republic of Iran
}

\author{
F. Zayeri, ${ }^{1}$ A. Kazemnejad, ${ }^{2}$ M. Ganjali, ${ }^{3}$ G. Babaei ${ }^{4}$ and F. Nayeri ${ }^{5}$
}

$$
\begin{aligned}
& \text { معلّل حدوث انخفاض حرارة الوليد وعوامل اختطلرها في مستشفيات الإحالة في طهران، بجمهورية }
\end{aligned}
$$

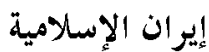

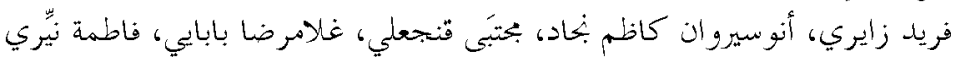

ABSTRACT To identify the incidence rate and risk factors of neonatal hypothermia at referral hospitals in Tehran, Islamic Republic of Iran, 900 neonates were randomly selected. Body temperature was measured repeatedly at different time points after birth. More than $50 \%$ became hypothermic soon after birth. Multiple regression analysis showed that low birth weight, low gestational age, low environmental temperature, low Apgar score, multiple pregnancy and receiving cardiopulmonary resuscitation were significantly associated with hypothermia. These findings suggested that there is an urgent need to sensitize and educate all levels of staff dealing with neonates in our country.

Incidence et facteurs de risque de l'hypothermie néonatale dans les hôpitaux de recours de Téhéran (République islamique d'Iran)

RÉSUMÉ Afin d'identifier l'incidence et les facteurs de risque de l'hypothermie néonatale dans les hôpitaux de recours de Téhéran (République islamique d'Iran), il a été procédé à la sélection randomisée de 900 nouveau-nés. Leur température corporelle a été mesurée à plusieurs reprises à différents points-temps après la naissance. Plus de $50 \%$ de ces nouveau-nés se sont révélés hypothermiques peu de temps après leur naissance. L'analyse de régression multiple a mis en évidence l'existence d'une association significative entre l'hypothermie et un faible poids de naissance, la prématurité, une température ambiante basse, un score d'Apgar faible, la multiparité et le recours à la réanimation cardiorespiratoire. Ces résultats semblent indiquer combien il est urgent dans notre pays de sensibiliser à ce problème et de former, à tous les échelons de la hiérarchie, les personnels amenés à prendre en charge les nouveau-nés.

${ }^{1}$ Department of Biostatistics, Faculty of Paramedical Sciences; ${ }^{3}$ Department of Statistics, Faculty of Mathematical Sciences, Shahid Beheshti University, Tehran, Islamic Republic of Iran.

${ }^{2}$ Department of Biostatistics, School of Medical Sciences, ${ }^{4}$ Tarbiat Modares University, Tehran, Islamic Republic of Iran (Correspondence to A. Kazemnejad; akili@yahoo.com).

${ }^{5}$ Department of Neonatology, Tehran University of Medical Sciences, Tehran, Islamic Republic of Iran.

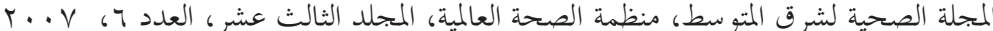




\section{Introduction}

Hypothermia is an important determinant of the survival of newborns, especially among low-birth-weight (LBW) babies [1]. An infant loses heat by evaporation, convection, radiation and conduction. If hypothermia persists, there is a risk of neonatal cold injury, in which case the infant usually becomes lethargic, with slow, shallow and irregular respiration and a slow heart rate corresponding to decreased body temperature. Prolonged cold injury leads to oedema, scleroderma, general haemorrhage (especially pulmonary haemorrhage), jaundice and death [2].

In many parts of the world, health personnel are not aware of the importance of keeping babies warm by simple methods such as drying and wrapping immediately after birth, avoiding harmful traditional practices, encouraging early breastfeeding and keeping newborns in close contact with their mothers [3]. A study on 160 medical and paramedical staff dealing with neonatal care in India showed that only $47.8 \%$ of the subjects defined neonatal hypothermia correctly. In addition, only $18.6 \%$ of the interviewees had knowledge about the correct method of recording the temperature in a newborn [4]. Previous reports from different Asian and African developing countries show that most of the neonates became hypothermic soon after birth [3]. In developed countries, however, awareness of the problem has resulted in improved care, and the incidence of neonatal hypothermia was mostly confined to outborn, premature and LBW infants.

In previous decades, most of the studies from countries of the Eastern Mediterranean Region focused on the prevention of neonatal hypothermia or its related complications among newborn infants. Therefore, adequate information about the prevalence of this problem is not available in the majority of these countries. For instance, a study on 50 Iraqi children with hypothermia showed that the majority of infants had evidence of infection, particularly septicaemia [5]. The overall mortality rate in that study was $26 \%$ (42\% in LBW infants) and the most common finding was a high incidence of aspiration pneumonia in infants over 3 days old. The results from another study on 36 cases of neonatal intestinal obstruction in Iraq revealed that hypothermia was one of the main causes of death [6]. In a survey of paediatric mortality in Lebanon, the researchers recommended that prevention of 5 risk factors-acidosis, hypoxaemia, hypoglycaemia, hypotension and hypothermia-was important for reducing the death rate [7]. Neonatal hypothermia is also prevalent in Israel [8]. Different studies in that country have shown that mortality and morbidity in hypothermic infants are mainly related to the presence or absence of an associated septicaemia [9-11]. In Turkey, a study on 66 babies who had undergone surgery because of peritonitis showed $100 \%$ mortality in hypothermic neonates [12].

In our country, Islamic Republic of Iran, previous reports about the prevalence and risk factors of neonatal hypothermia are confined to unpublished studies, small sample sizes or local surveys. Regarding this variable information, we believe that neonatal hypothermia is a serious health problem in our country, even among those born at university teaching hospitals. Therefore, we decided to design an epidemiologic survey to obtain more accurate information about the incidence rate and risk factors of this problem at referral university teaching hospitals of Tehran, which are expected to have better trained staff as well as higher level of medical resources compared to small or local centres in other cities of the Islamic Republic of Iran.

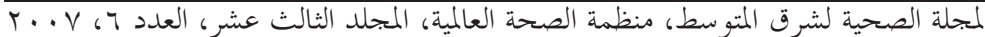


Here, it should be noted that while other surveys in the area of neonatal hypothermia have generally focused on its incidence, our study gives more attention to the severity of hypothermia through a longitudinal study. This repeated measures study helps us to assess the trend of improvement among hypothermic infants after recommended treatments. The results would provide a baseline for future health programmes in these hospitals.

\section{Methods}

\section{Sampling technique}

This study of neonatal hypothermia was an epidemiologic longitudinal survey at referral university hospitals in Tehran, Islamic Republic of Iran. Since one of the most important objectives of the study was to estimate the prevalence of neonatal hypothermia at these hospitals, the sample size formula for the prevalence studies $(n=$ $\left.Z^{2} P(1-P) / d^{2}\right)$, with $\alpha=0.02, d=0.04$ and $P=0.5$ (which yields the maximum sample size), was used to estimate the required sample size. Using simple calculation, it was found that the study sample should consist of at least 845 newborns.

There are 15 referral university teaching hospitals with a neonatal intensive care unit (NICU) in Tehran. To select the study sample, the city was divided into 5 districts (north, south, east, west and centre), each with 3 referral hospitals. Then 1 hospital was randomly selected in each district. In the next stage, 100 days were randomly chosen between February and November 2004 (to include 4 seasons with air temperature ranging from $-5^{\circ} \mathrm{C}$ to $40{ }^{\circ} \mathrm{C}$ ). Finally, for each selected day, 2 newborn infants were selected at each hospital using a random numbers table. After excluding hyperthermic neonates (rectal temperature
$>38{ }^{\circ} \mathrm{C}$ ), outborn babies (because of inadequate sample size) and those with diagnosable anomalies at birth, 900 neonates were recruited to the study.

\section{Repeated measurements}

After obtaining consent from the parents, the neonate's rectal temperature was measured using a calibrated digital low-reading thermometer at 5 time periods: immediately after birth in the operating room, several minutes after admission to the neonatal unit (levels I, II, III of nursery care), and 1, 2, and 4 hours after admission to the neonatal unit. If a newborn was hypothermic, she/ he was rewarmed according to the World Health Organization recommendations [3]. The outcome variable for each newborn was the severity of hypothermia, graded as follows: normal body temperature (rectal temperature $36.5-38.0^{\circ} \mathrm{C}$ ); mild hypothermia (rectal temperature $35-36.5^{\circ} \mathrm{C}$ ); moderate hypothermia (rectal temperature $32-35^{\circ} \mathrm{C}$ ); or severe hypothermia (rectal temperature $<32{ }^{\circ} \mathrm{C}$ ).

At each hospital, body temperatures of the neonates were measured by 2 trained nurses. Since the body temperature for each baby was considered an ordinal outcome (severity of hypothermia according to the classification), the inter-observer and intraobserver reliability were assessed via a pilot study using generalized Kappa index [13]. All the obtained inter- and intra-observer Kappa statistics were about $100 \%$ for these nurses. These findings indicated perfect inter- and intra-observer reliability in this study.

\section{Risk factors}

After preliminary analyses, the following explanatory variables were considered potential risk factors for neonatal hypothermia in the regression analysis: sex $(0=$ male, 1 
= female), birth weight $(0=\geq 2500 \mathrm{~g}, 1=$ $<2500$ g), gestational age $(0=\geq 37$ weeks, $1=<37$ weeks), environmental temperature (temperature of the operating room and neonatal unit), Apgar score $(0=\geq 8,1$ $=<8)$, pregnancy type $(0=$ singleton, $1=$ multiple) and cardiopulmonary resuscitation $(\mathrm{CPR})(0=$ not received, $1=$ received $)$. Note that, sex, birth weight, gestational age, pregnancy type and CPR variables are time-stationary factors (which are constant at different time points of the study), but environmental temperature and Apgar score are time-dependent variables (which may vary at different time points).

\section{Statistical analysis}

The descriptive part of the statistical analysis was carried out using rates and frequency tables. For analytical purposes, a multivariate logistic regression model (marginal model) was utilized. The generalized estimating equations methodology was also used for estimating the regression parameters and accounting for the correlation between repeated outcomes. The analysis was performed using the Genmod procedure in $S A S$ software, version 8. $P$-values less than 0.05 were considered statistically significant.

\section{Results}

\section{Description of the data}

The study sample consisted of 900 neonates (452 males and 448 females). Of these, $298(33.1 \%)$ had birth weight $<2500 \mathrm{~g}$, and $323(35.9 \%)$ were preterm (gestational age $<37$ weeks). The mean temperature of the operating rooms and neonatal units at these hospitals was about $28.5^{\circ} \mathrm{C}$ (standard deviation $=1.7)$. In addition, 174 neonates $(19.3 \%)$ had Apgar score $<8$ on at least one occasion. In this sample, the rate of multiple pregnancy was about $3.5 \%$. Additionally, 104 neonates $(11.6 \%)$ received CPR during the study.

Table 1 shows the severity of hypothermia among these babies at different time points. Summing the data for mild, moderate and severe hypothermia shows that $53.3 \%$ were hypothermic immediately after birth, $13.6 \%$ on admission to the NICU, $2.7 \% 1$ hour after admission, $0.5 \% 2$ hours after admission and $0.3 \% 4$ hours after admission.

\section{Analysis of risk factors and death rate}

A multivariate logistic regression model was utilized to identify some of the most

\begin{tabular}{|c|c|c|c|c|c|c|c|c|}
\hline \multirow[t]{3}{*}{ Time } & \multicolumn{8}{|c|}{ Severity of hypothermia } \\
\hline & \multicolumn{2}{|c|}{ Normal } & \multicolumn{2}{|c|}{ Mild } & \multicolumn{2}{|c|}{ Moderate } & \multicolumn{2}{|c|}{ Severe } \\
\hline & No. & $\%$ & No. & $\%$ & No. & $\%$ & No. & $\%$ \\
\hline Immediately after birth & 420 & 46.7 & 426 & 47.3 & 50 & 5.6 & 4 & 0.4 \\
\hline At admission to NICU & 778 & 86.4 & 114 & 12.7 & 5 & 0.6 & 3 & 0.3 \\
\hline $1 \mathrm{~h}$ after admission & 875 & 97.2 & 21 & 2.3 & 2 & 0.2 & 2 & 0.2 \\
\hline $2 \mathrm{~h}$ after admission & 895 & 99.4 & 3 & 0.3 & 2 & 0.2 & 0 & 0.0 \\
\hline $4 \mathrm{~h}$ after admission & 897 & 99.7 & 2 & 0.2 & 1 & 0.1 & 0 & 0.0 \\
\hline
\end{tabular}

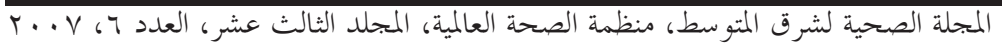


important factors associated with neonatal hypothermia at these hospitals. To do this, the mild, moderate and severe categories of hypothermia were combined at different occasions in order to obtain a repeated binary response. In the regression analysis, thus, the repeated response data for each newborn can be written as: $0=$ normothermic (body temperature $\left.36.5-38^{\circ} \mathrm{C}\right), 1=$ hypothermic (body temperature $<36.5^{\circ} \mathrm{C}$ ), at different time periods.

Table 2 shows the results of multiple regression analysis for the illustrated model. These estimates show that all the described factors except sex of neonate were significantly associated with neonatal hypothermia. In other words, infants who were LBW, preterm, of low Apgar score, from multiple pregnancies and received CPR had higher risk of being hypothermic. Another notable result of regression analysis was that their body temperature was significantly related to the environmental temperature $(P<0.001)$. The neonates had higher risk for being hypothermic when the operating room or neonatal unit temperature was lower.

In this regression analysis, interpretation of the estimates may be more comprehensible in terms of the odds ratios (OR). For example, since the estimate of gestational age effect is 0.549 , it can be concluded that preterm neonates had $\mathrm{OR}=\exp (0.549)=$ 1.73 times the odds of being hypothermic compared to term neonates. In addition, the estimate of environmental temperature

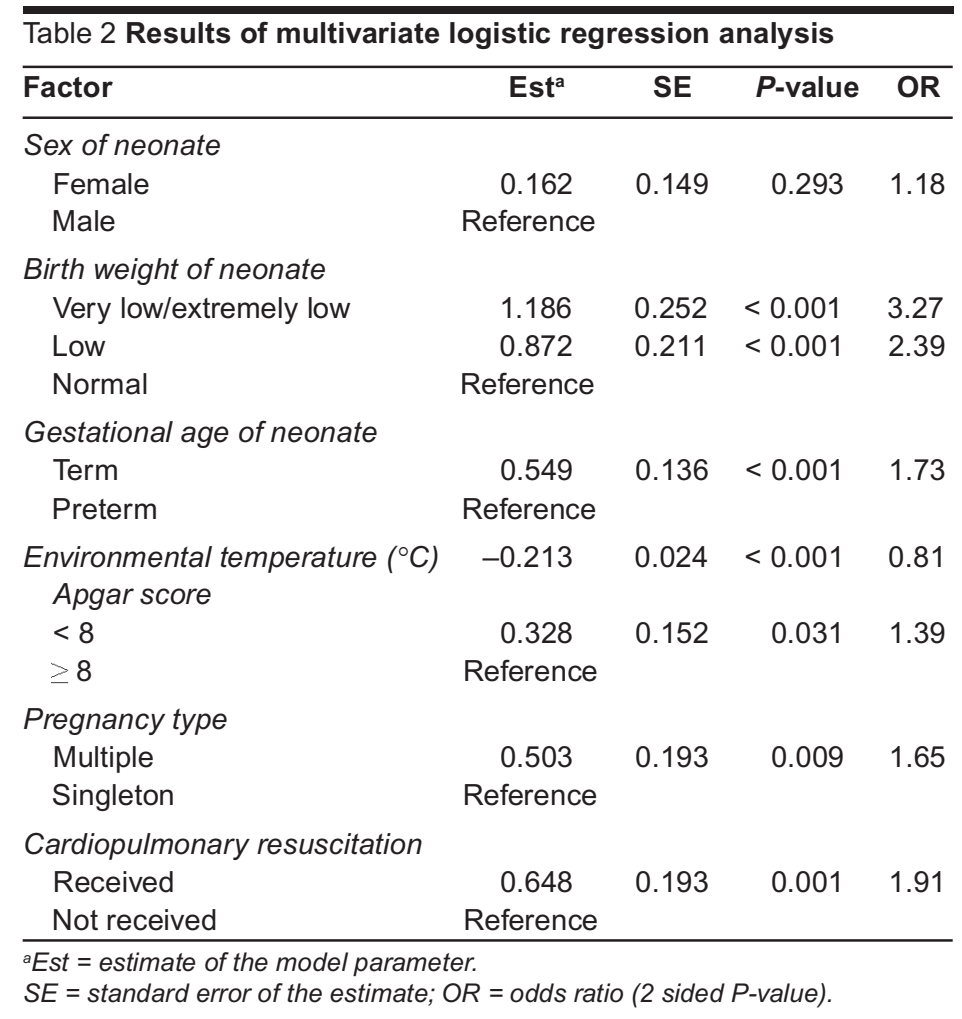

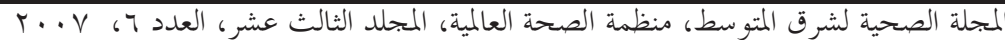


effect $(-0.213)$ tells us that a $1{ }^{\circ} \mathrm{C}$ increase in the operating room or neonatal unit temperature decreases the odds of neonatal hypothermia by exp $(-0.213)=0.81$.

In order to provide more detailed information about the relationship between severity of hypothermia and the significant factors, we classified the severity of hypothermia immediately after admission to the neonatal unit in terms of weight, gestational age, environmental temperature, Apgar score, type of pregnancy and CPR. Table 3 shows the results. It is clear that the mild, moderate and severe hypothermia in infants who were LBW, preterm, of low
Apgar score, from multiple pregnancies and received CPR were significantly more prevalent compared with infants who were not LBW, term, of normal Apgar, singleton and those not received CPR. For instance, the prevalence of mild, moderate and severe hypothermia in low Apgar score infants were respectively $35.2 \%, 3.7 \%$ and $5.5 \%$, while these rates were $11.2 \%, 0.4 \%$ and $0.0 \%$ in normal Apgar score babies.

Finally, it is important to note that total mortality rate was 6\% (54 neonates) during this study and the death rate in hypothermic babies was considerably higher than in normothermic ones. Table 4 shows the death

\begin{tabular}{|c|c|c|c|c|c|}
\hline \multirow[t]{2}{*}{ Factor } & \multirow{2}{*}{$\begin{array}{c}\text { Total } \\
\text { No. }\end{array}$} & \multicolumn{3}{|c|}{ Severity of hypothermia } & \multirow[b]{2}{*}{$\begin{array}{c}\text { Severe } \\
\%\end{array}$} \\
\hline & & $\begin{array}{l}\text { Normal } \\
\%\end{array}$ & $\underset{\%}{\text { Mild }}$ & $\begin{array}{c}\text { Moderate } \\
\%\end{array}$ & \\
\hline \multicolumn{6}{|l|}{ Weight $(g)$} \\
\hline$<1000$ & 26 & 69.7 & 21.2 & 6.1 & 3.0 \\
\hline $1000-<1500$ & 65 & 69.8 & 28.6 & 1.6 & 0.0 \\
\hline $1500-<2500$ & 207 & 78.8 & 20.3 & 0.9 & 0.0 \\
\hline$\geq 2500$ & 602 & 92.2 & 7.5 & 0.0 & 0.3 \\
\hline \multicolumn{6}{|l|}{ Gestational age } \\
\hline Preterm & 323 & 79.0 & 19.5 & 1.2 & 0.3 \\
\hline Term & 577 & 90.6 & 8.9 & 0.2 & 0.3 \\
\hline \multicolumn{6}{|l|}{ Apgar score } \\
\hline$<8$ & 174 & 55.6 & 35.2 & 3.7 & 5.5 \\
\hline$\geq 8$ & 726 & 88.4 & 11.2 & 0.4 & 0.0 \\
\hline \multicolumn{6}{|l|}{ Pregnancy type } \\
\hline Multiple & $\begin{array}{r}32 \\
\text { (labour) }\end{array}$ & 73.4 & 25.0 & 1.6 & 0.0 \\
\hline Single & 834 & 87.7 & 11.6 & 0.5 & 0.2 \\
\hline \multicolumn{6}{|l|}{$\begin{array}{l}\text { Cardiopulmonary } \\
\text { resuscitation }\end{array}$} \\
\hline Received & 104 & 71.2 & 26.0 & 1.9 & 1.0 \\
\hline Not received & 796 & 88.4 & 10.9 & 0.4 & 0.3 \\
\hline $\begin{array}{l}\text { Environmental } \\
\text { temperature }{ }^{\circ} C^{a}\end{array}$ & 898 & $29.2(1.7)$ & $28.1(1.4)$ & $27.8(1.9)$ & $27.5(2.0)$ \\
\hline
\end{tabular}

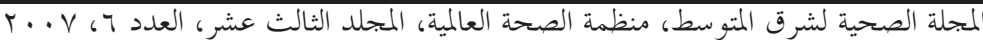




\begin{tabular}{|c|c|c|c|c|c|c|c|}
\hline \multirow[t]{3}{*}{ Time } & \multicolumn{6}{|c|}{ Severity of hypothermia } & \multirow{3}{*}{$P$-value } \\
\hline & \multirow{2}{*}{$\begin{array}{l}\text { Normal } \\
\text { No. dead/ } \\
\text { total }\end{array}$} & \multirow{2}{*}{\multicolumn{2}{|c|}{$\begin{array}{ll}\text { No. dead } / \\
\text { total }\end{array}$}} & \multicolumn{3}{|c|}{ Moderate/severe } & \\
\hline & & & & $\%$ & $\begin{array}{l}\text { No. dead/ } \\
\text { total }\end{array}$ & $\%$ & \\
\hline Immediately after birth & $11 / 420$ & 2.6 & $31 / 426$ & 7.3 & $12 / 54$ & 22.2 & $<0.001$ \\
\hline At admission to NICU & $35 / 778$ & 4.5 & $16 / 114$ & 14.0 & $3 / 8$ & 37.5 & $<0.001$ \\
\hline $1 \mathrm{~h}$ after admission & $50 / 875$ & 5.7 & $2 / 21$ & 9.5 & $2 / 4$ & 50.0 & 0.001 \\
\hline $2 \mathrm{~h}$ after admission & $52 / 895$ & 5.8 & $1 / 3$ & 33.3 & $1 / 2$ & 50.0 & 0.004 \\
\hline
\end{tabular}

NICU = neonatal intensive care unit.

rate by severity of hypothermia at different time periods. The results of chi-squared tests showed a significant relation between neonatal death and hypothermia (because of inadequate sample size for chi-squared test, the last time period was not shown in this table).

\section{Discussion}

Hypothermia is an important and independent risk factor for neonatal death [14]. In addition, as indicated in previous studies, hypothermia is one the most important cause of neonatal morbidity. For instance, it has been shown that hypothermia is associated with neonatal complications such as pulmonary haemorrhage, acidaemia, scleroderma, jaundice and hypoglycaemia [1,3,14-18].

To control this health problem and its related complications, prevention is preferable to treatment. In this context, implementing WHO practical guides for thermal control of newborns is a helpful strategy for reducing the incidence of this problem in developing countries [3]. Among the proposed preventive methods, however, "kangaroo" mother care (where the preterm infants are carried skin-to-skin with the mother) is the only effective, affordable and available method in most developing countries [19], especially in rural areas.

Several traditional and modern methods have been previously suggested for the diagnosis of neonatal hypothermia. For instance, Singh et al. suggested a simple method for the assessment of a newborn baby's temperature by human touch [20]. Using this method, mothers, traditional birth attendants, nurses and physicians can successfully undergo training to assess a newborn's temperature by touching the abdomen and feet. However, proper diagnosis usually requires a low-reading thermometer, lacking which, the diagnosis can be suspected but is often missed [11].

Methods of thermal control and managing hypothermic infants are strongly related to the traditional beliefs and medical resources in each country. A variety of articles are available from different countries about encouraging or discouraging the traditional or other commonly used methods. For instance, in developed countries, highly sophisticated incubators and radiant warmers are available, but there is increasing concern about the long-term effects of noise and light disturbance and unplanned procedures associated with the use of these tools [19]. In Malaysia, cleaning newborn infants with coconut oil shortly after birth is a com- 
mon practice in labour rooms. A study on 227 randomly selected normal-term infants in this country revealed that this traditional method of cleaning is significantly associated with hypothermia [21]. The researchers concluded that the labour room temperature should be set at a higher level and cleaning infants using this method should be discouraged. In the Himalayan state of north India, a warm heated room for delivery and lyingin, early rooming in, oil massage and layers of warm clothing are traditional means for thermo-regulation [22]. In Turkey, Sarman et al. randomly assigned 60 LBW hypothermic newborns admitted to a neonatal care unit for treatment either in a cot on a heated, water-filled mattress kept at $37^{\circ} \mathrm{C}$ or in an air-heated incubator with a mean temperature of $35^{\circ} \mathrm{C}$. They demonstrated that normal temperatures were achieved within the first day and remained within this range during the subsequent days after admission in all the infants treated on the mattress, whereas they were not achieved until 3 days later in the incubator group. The neonatal mortality among those treated on a mattress was $21 \%$ and among those treated in the incubator $34 \%$. They concluded that a heated, water-filled mattress provides a good alternative to skin-to-skin contact with the mother, and to the use of a complex and expensive incubator for rapidly attaining and maintaining normal temperatures in the LBW newborn [23].

In developed countries, the incidence of neonatal hypothermia is mostly confined to LBW and small-for-gestationalage neonates. Therefore, the majority of studies have been focused on LBW, premature and other high-risk neonates. For instance, a study on very-low-birth-weight (VLBW) infants in Canada showed that the overall incidence of moderate to severe hypothermia (body temperature $<35{ }^{\circ} \mathrm{C}$ ) on admission was $11.5 \%-12.5 \%$ among neonates [15]. In a retrospective study in USA, it was revealed that $45 \%$ of outborn VLBW infants were hypothermic (body temperature $<36.3^{\circ} \mathrm{C}$ ) on admission [17]. Another study, in Australia, showed that $17 \%$ of infants born during transport were hypothermic (axillary temperature less than $36{ }^{\circ} \mathrm{C}$ ) [24]. In the United Kingdom, a study on 4004 infants born before 26 weeks of gestation showed that among those admitted for intensive care, $66.7 \%, 80.0 \%$, $58.3 \%, 42.7 \%$ and $29.6 \%$ were hypothermic at weeks 21, 22, 23, 24 and 25 of gestational age respectively [25].

In developing countries, however, this problem is more prevalent even in healthy full term and normal-birth-weight (NBW) infants. In Nepal, a study on 500 inborns revealed that $85 \%$ of neonates were hypothermic (body temperature $<36{ }^{\circ} \mathrm{C}$ ) 2 hours after delivery [26]. In north India, research on 189 term healthy neonates delivered at home showed hypothermia in $19.1 \%$ and $3.1 \%$ respectively in winter and summer, 24 hours after delivery (axillary temperature $<35.6^{\circ} \mathrm{C}$ ) [27]. In our study, $7.8 \%$ of NBWs and $9.4 \%$ of full-term babies were hypothermic after admission to the neonatal unit. Hypothermia is a serious health problem, even in tropical developing countries, despite warm environmental conditions, and it contributes to a high neonatal morbidity and mortality. A study on neonates admitted to a neonatal care unit in Tanzania revealed that $22.4 \%$ were hypothermic (axillary temperature $<35.6{ }^{\circ} \mathrm{C}$ ). Severe hypothermia was found in $13 \%$ of these neonates (axillary temperature $<32{ }^{\circ} \mathrm{C}$ ) and hypothermic infants had a 3-fold higher mortality and morbidity [28]. In another study on 313 consecutive newborn infants admitted to a neonatal unit in Harare, Zimbabwe, it was found that the prevalence of hypothermia on admission was $85 \%$, with a mean axillary temperature of $34.3^{\circ} \mathrm{C}$ [29]. In Zambia, a

المجلة الصحية لشرق المتو سط، منظمة الصحة العالمية، المجلد الثالث عشر، العدد ب، V... 
prospective study at a university teaching hospital among 261 infants aged $0-7$ days admitted to the paediatric unit during the warm season revealed that $44 \%$ were hypothermic (body temperature $<36{ }^{\circ} \mathrm{C}$ ) on admission [30].

In the present study, $33.1 \%$ of infants were of LBW, $35.9 \%$ were premature and 19.3\% had low Apgar scores. It should be noted that the selected hospitals are referral tertiary care centres, so a considerable proportion of high-risk pregnant women are referred to these centres from different parts of the country. This is the possible reason for the high rate of LBW, low Apgar score and preterm neonates.

The results of logistic regression analysis showed that infants who were LBW, premature, with low Apgar score, from a multiple pregnancy and had received CPR had higher risk of being hypothermic. Our study did not show any relation between hypothermia and sex of neonate. Kambarami and Chidede did not find any significant relationship between need for resuscitation, birth weight, sex and neonatal hypothermia [29]. However, recent studies from different parts of the world have shown a significant association between neonatal hypothermia and LBW, prematurity, low Apgar sore, deliveries from outside hospital, inadequate clothing after delivery, low socioeconomic status of the mother and air temperature [8,28,30-32]. Additionally, our study showed a strong correlation between environmental temperature and neonatal hypothermia. Kumar and Aggarwal reported a significant correlation between room air temperature and neonatal temperature among home-delivered newborns in north India [27]. In addition, Cheah and Boo found that lower labour room temperature was a significant risk factor for being hypothermic [21].

The above mentioned findings help us to sensitize and educate all levels of staff dealing with neonates about the risk factors of neonatal hypothermia in developing countries. Proper management of LBW and preterm neonates is of great importance to reduce the prevalence of hypothermia and subsequent problems among newborn infants. Recently, major studies have been undertaken about the management and outcomes of extremely preterm neonates in Sweden and the United Kingdom [25,3336].

In conclusion, it should be stated that the effect of hypothermia on neonatal mortality and morbidity is undeniable $[7,14,30,37]$. The results from the present study showed that more than $50 \%$ of the infants suffered from hypothermia soon after birth at referral university teaching hospitals of Tehran. This concerning result shows that the extent and significance of neonatal hypothermia are not fully realized in our country. Here it should be emphasized that the nursing staff play a vital role for controlling this health problem in developing countries such as the Islamic Republic of Iran. Therefore, there is an urgent need to increase awareness about the consequences of hypothermia and train mothers and all levels of neonatal care staff to control this health problem in developing countries.

\section{References}

1. Vobra $S$ et al. Effect of polyethylene occlusive skin wrapping on heat loss in very low birth weight infant at delivery: a randomized trial. Journal of pediatrics, 1999, 134:547-51.
2. LeBlanc $\mathrm{MH}$. The physical environment. In: Fanaroff A, Martin RJ, eds. Neonatalperinatal medicine, 7 th ed. Volume 1. St Louis, Missouri, Mosby, 2002:512-24. 
3. Thermal control of the newborn: a practical guide. Geneva, World Health Organization, 1993 (WHO/FHF/SM).

4. Choudhary SP, Bajaj RK, Gupta RK. Knowledge, attitude and practices about neonatal hypothermia among medical and paramedical staff. Indian journal of pediatrics, 2000, 67:491-6.

5. El-Radhi AS, Al-Kafaji N. Neonatal hypothermia in a developing country. Clinical pediatrics, 1980, 19:401-4.

6. Nasir GA, Rahma S, Kadim AH. Neonatal intestinal obstruction. Eastern Mediterranean health journal, 2000, 6:187-93.

7. Mounla NA, Khudr AA. Pediatric mortality: an avoidable tragedy. Le Journal médical libanais, 1989, 38:25-8.

8. Zabelle $\mathrm{J}$ et al. Risk factors for infantile hypothermia in early neonatal life. Pediatric emergency care, 1990, 6:96-8.

9. Sofer S, Benkovich E. Severe infantile hypothermia: short- and long-term outcome. Intensive care medicine, 2000, 26:88-92.

10. Dagan R, Gorodischer R. Infections in hypothermic infants younger than 3 months old. American journal of diseases of children, 1984, 138:483-5.

11. Goldsmith JR, Arbeli Y, Stone D. Preventability of neonatal cold injury and its contribution to neonatal mortality. Environmental health perspectives, 1991, 94:55-9.

12. Zurlodemir $U$ et al. Neonatal peritonitis. Turkish journal of pediatrics, 1992 , 34:157-66.

13. Agresti A. Categorical data analysis. New York, John Wiley and Sons, 1990, 34785.

14. Da Mota Silveira SM et al. Hypothermia on admission: a risk factor for death in newborns referred to the Pernambuco Institute of Mother and Child Health. Journal of tropical pediatrics, 2003, 49:115-20.
15. Hazan J, Maag U, Chessex P. Association between hypothermia and mortality rate of premature infants-revisited. American journal of obstetrics and gynecology, 1991, 164:111-2.

16. Johanson RB et al. A survey of technology and temperature control on a neonatal unit in Katmandu, Nepal. Journal of tropical pediatrics, 1993, 39:4-10.

17. Loughead MK, Loughead JL, Reinhart MJ. Incidence and physiologic characteristics of hypothermia in the very low birth weight infant. Pediatric nursing, 1997, 23:11-5.

18. Xiao-Cheng J, Chuan-You Z, Ru-Yan P. Epidemiologic study on hypothermia in newborns. Chinese medical journal, 1993 10:428-32.

19. Tunell R. Prevention of neonatal cold injury in preterm infants. Acta paediatrica, 2004, 308-10.

20. Sing M, Rao G, Malhotra AK. Assessment of newborn baby's temperature by human touch: a potentially useful primary care strategy. Indian pediatrics, 1992, 29:449-52.

21. Cheah FC, Boo NY. Risk factors associated with neonatal hypothermia during cleaning of newborn infants in labour room. Journal of tropical pediatrics, 2000 , $46: 46-50$.

22. lyengar SD, Bhakoo ON. Prevention of neonatal hypothermia in Himalayan villages. Role of the domiciliary caretaker. Tropical and geographical medicine, 1991, 43:293-6.

23. Sarman I, Can G, Tunell R. Rewarming preterm infants on a heated, water filled mattress. Archives of disease in childhood, 1989, 64:687-92.

24. Bowman Ed, Ray RN. Control of temperature during newborn transport: an old problem with new difficulties. Journal 
of paediatrics and child health, 1997, 33:398-401.

25. Costeloe $\mathrm{K}$ et al. The EPICure study: outcomes to discharge from hospital for infants born at the threshold of viability. Pediatrics, 2000, 106:659-71.

26. Johanson RB et al. Effect of post-delivery care on neonatal body temperature. Acta paediatrica, 1992, 81:859-63.

27. Kumar R, Aggarwal AK. Body temperature of home delivered newborns in north India. Tropical doctor, 1998, 28:134-6.

28. Manji KP, Kisenge R. Neonatal hypothermia on admission to a special care unit in Dar-es-Salam, Tanzania: a cause for concern. Central African journal of medicine, 2003, 49:23-7.

29. Kambarami R, Chidede O. Neonatal hypothermia levels and risk factors for mortality in a tropical country. Central African journal of medicine, 2003, 49:103-6.

30. Christensson $\mathrm{K}$ et al. The effect of routine hospital care on the health of hypothermic newborn infants in Zambia. Journal of tropical pediatrics, 1995, 41:210-4.

31. Ondoa-Onama C, Tumwine JK. Immediate outcome of babies with low Apgar score in Mulago Hospital, Uganda. East African medical journal, 2003, 80:22-9.

32. Doctor BA et al. Perinatal correlates and neonatal outcomes of small for gesta- tional age infants born at term gestation. American journal of obstetrics and gynecology, 2001, 185:652-9.

33. Wood NS et al. The EPICure study: growth and associated problems in children born at 25 weeks of gestational age or less. Archives of disease in childhood. Fetal and neonatal edition, 2003, 88:492-500.

34. Serenius F et al. Short-term outcome after active perinatal management at 23-25 weeks of gestation. A study from two Swedish tertiary care centers. Part 1: maternal and obstetric factors. Acta paediatrica, 2004, 93:945-53.

35. Serenius F et al. Short-term outcome after active perinatal management at 23-25 weeks of gestation. A study from two Swedish tertiary care centers. Part 2 infant survival. Acta paediatrica, 2004, 93:1081-9.

36. Serenius $F$ et al. Short-term outcome after active perinatal management at 23-25 weeks of gestation. A study from two Swedish tertiary care centers. Part 3: neonatal morbidity. Acta paediatrica, 2004, 93:1090-7.

37. Daga AS, Daga SR, Patole SK. Determinants of death among admission to intensive care unit for newborns. Journal of tropical pediatrics, 1991, 37:53-6. 